\title{
Retorno al ejercicio después de COVID-19. Posicionamiento de la Sociedad Mexicana de Cardiología
}

\author{
Return to exercise after COVID-19. Statement of the Mexican Society of Cardiology
}

Norma Cerón-Enriquez ${ }^{*}$, Marianna A. García-Saldivia ${ }^{2}$, Jorge A. Lara-Vargas ${ }^{3}$, Juan P. Núñez-Urquiza J. Jesús Alonso-Sánchez ${ }^{5}$, J. Jesús Silva-Torres ${ }^{6}$, Juan C. Pérez-Gámez ${ }^{7}$, Nancy Pacheco-Beltrán ${ }^{8}$

y Marco A. Alcocer-Gamba ${ }^{9}$

${ }^{1}$ Unidad de Rehabilitación Cardiopulmonar, Centro Cardiopulmonar, Hospital Ángeles Puebla, Puebla, Pue.; ${ }^{2}$ Servicio de Cardiología, Unidad de Rehabilitación Cardiovascular, Hospital Regional de Alta Especialidad de Ixtapaluca, Ixtapaluca; ${ }^{3}$ Servicio de Rehabilitación Cardiaca, Departamento de Cardiocirugía, Centro Médico Nacional 20 de Noviembre, Ciudad de México; ${ }^{4}$ Departamento de Rehabilitación Cardiaca, Instituto del Corazón de Querétaro, Querétaro, Qro.; ${ }^{5}$ Servicio de Rehabilitación Cardiaca, Departamento de Cardiología, Hospital Mac Bernardette, Guadalajara, Jal.; ${ }^{6}$ Servicio de Cardiología Preventiva y Rehabilitación Cardiaca del Centro Médico Nacional Siglo XXI, Instituto Mexicano del Seguro Social, Ciudad de México; ${ }^{7}$ Servicio de Rehabilitación Cardiopulmonar, Hospital de Cardiología de la Unidad Médica de Alta Especialidad n.․ㅜ 34, Instituto Mexicano del Seguro Social, Monterrey, N.L.; ${ }^{8}$ Servicio de Cardiología, Instituto de Seguridad Social y Servicios Sociales de los Trabajadores del Estado, Querétaro, Qro; ${ }^{9}$ Laboratorio de Hemodinámica y Cardiología lintervencionista, Hospital Angeles Querétaro, Querétaro, Qro., México

\begin{abstract}
Resumen
La enfermedad por coronavirus 2019 (COVID-19) fue declarada pandemia el 11 de marzo de 2020; una consecuencia ha sido el incremento en el sedentarismo y la reducción de la actividad deportiva. El ejercicio beneficia el sistema inmunitario de defensa, especialmente en adultos mayores. Se recomienda guardar distancia de 1.5 metros entre personas, y si se realiza caminata o trote, el espacio debe ser de hasta 5 y 10 metros respectivamente. Los casos reportados son en su mayoría leves hasta en un $80 \%$, y pueden ser críticos hasta en 4.7\%; los factores de riesgo son bien conocidos: hipertensión, diabetes y enfermedad cardiaca previa. Los casos graves o críticos se presentan como cuadros de síndrome de distrés respiratorio agudo y ante afección cardiovascular cursan principalmente como miopericarditis, síndromes coronarios agudos, choque cardiogénico y eventos trombóticos, entre otros. El ejercicio después de la recuperación de infección por coronavirus 2 del síndrome respiratorio agudo grave (SARS-CoV-2) siempre está recomendado, sin embargo, dependerá del cuadro clínico qué medidas se deben tomar previo a su inicio, y es en casos moderados y especialmente en los graves donde la evaluación y prescripción previa al retorno al ejercicio o deporte debe ser guiada por personal médico experto en rehabilitación cardiopulmonar, en especial en deportistas.
\end{abstract}

Palabras clave: Ejercicio. COVID-19. Rehabilitación cardiaca. Miocarditis. Actividad física. Deporte.

\begin{abstract}
The coronavirus disease 2019 (COVID-19) was declared a pandemic on March 11, 2020; one consequence has been the increase in sedentary lifestyle and reduction of sports activity. Exercise benefits the immune defense system especially in
\end{abstract}

\section{Correspondencia:}

*Norma Cerón-Enríquez

E-mail: normahceron@yahoo.com
Disponible en internet: 01-03-2021 Arch Cardiol Mex. 2021;91(Supl):102-109 www.archivoscardiologia.com

1405-9940 / @ 2021 Instituto Nacional de Cardiología Ignacio Chávez. Publicado por Permanyer. Este es un artículo open access bajo la licencia CC BY-NC-ND (http://creativecommons.org/licenses/by-nc-nd/4.0/). 
older adults; it is recommended to keep a distance of 1.5 meters between people, and if walking or jogging is carried out, the space must be up to 5 and 10 meters respectively. The reported cases are mostly mild up to $80 \%$ and can be critical in up to 4.7\%; the risk factors are well known, hypertension, diabetes and previous heart disease. Severe or critical cases present as symptoms of acute respiratory distress syndrome, and in the case of cardiovascular disease, they mainly occur as myopericarditis, acute coronary syndromes, cardiogenic shock, thrombotic events, among others. Returning to exercise after recovery from severe acute respiratory syndrome coronavirus 2 (SARS-CoV-2) infection is always recommended, however it will depend on the clinical picture what measures should be taken prior to its onset, and it is in moderate cases and especially in the severe ones where the evaluation and prescription prior to returning to exercise or sport should be guided by medical personnel experts in cardiopulmonary rehabilitation, especially in athletes.

Key words: Exercise. COVID-19. Cardiac rehabilitation. Myocarditis. Physical activity. Sports.

\section{Introducción}

La enfermedad por coronavirus 2019 (COVID-19), causante de síndrome de distrés respiratorio agudo (SDRA, SARS en inglés) y causada por la infección por coronavirus 2 del síndrome respiratorio agudo grave (SARSCoV-2), fue declarada pandemia el 11 de marzo de 2020. Una consecuencia ha sido el incremento del sedentarismo y la suspensión masiva del ejercicio y actividades deportivas. Lo anterior toma relevancia, ya que el ejercicio es un factor preventivo y terapéutico de muchas patologías de riesgo cardiovascular; su práctica regular a diferentes volúmenes beneficia el sistema inmunitario de defensa, reduce las citocinas proinflamatorias, incrementa la actividad citotóxica de las células natural killer y TCD8+, y mejora la función de los neutrófilos y la proliferación de linfocitos $\mathrm{B}^{1}$, especialmente en adultos mayores, generando un efecto protector contra virus y bacterias e incluso mejorando la respuesta inmunológica de la vacuna contra influenza ${ }^{2}$. Al realizarlo se enfatiza la necesidad de utilización de mascarilla ${ }^{3}$; un distanciamiento entre personas de 1.5 metros, 5 metros en caminata $(4 \mathrm{~km} / \mathrm{h})$ y 10 metros al correr $(14.4 \mathrm{~km} / \mathrm{h})^{4}$. Incluso se ha sugerido en cuarentena incrementar el volumen de ejercicio $^{5}$. Si este debe retomarse no es la duda, sin embargo, este virus ha demostrado ser altamente infeccioso, con un impacto cardiovascular que se documenta cada vez más en los casos recuperados ${ }^{6}$. El objetivo de este posicionamiento es repasar los efectos pulmonares y principalmente cardiovasculares de la COVID-19 y especialmente definir las prácticas más seguras del retorno al ejercicio, en población general y deportistas.

\section{COVID-19, descripción fisiopatológica: papel en el daño miocárdico y pulmonar}

El coronavirus 2 del síndrome respiratorio agudo grave (SARS-CoV-2) es miembro de la familia Coronaviridae, virus ARN de cadena sencilla, porta un receptor que se une a la enzima convertidora de angiotensina 2, altamente expresada en las células del alvéolo pulmonar y del miocardio; el grado de afección clínica se ha reportado de curso leve en el $81.4 \%$ de los casos, severo en el $13.9 \%$ y crítico en el $4.7 \%$. padecer la fase más grave de la infección se incrementa con edad mayor a 65 y con factores clásicos de riesgo como hipertensión, enfermedad cardio-cerebrovascular y diabetes, con una prevalencia reportada del $17.1,16.4$ y $9.7 \%$ respectivamente, además de mayor tasa de mortalidad (10.5\%) ante enfermedad cardiovascular confirmada ${ }^{9}$. Una posible causa es el daño miocárdico previo, encontrándose mayor prevalencia de este dato en aquellos que ingresaron a cuidados críti$\cos (22.2$ vs. $2 \% ; p<0.001)$, e igualmente entre pacientes que fallecieron (59 vs. $1 \% ; p<0.0001)^{10}$.

\section{Cuadro clínico leve, moderado y grave de la infección por SARS-CoV-2}

La infección por SARS-CoV-2 causa primordialmente síntomas respiratorios, pero también cuadros cardiovasculares graves, y las complicaciones puedan tener una morbilidad de seis meses y una fase crónica de seguimiento y rehabilitación de 12 meses o más. La gravedad clínica se ha clasificado en cuatro categorías: infectados asintomáticos, sintomáticos de estadía en casa, sintomáticos admitidos al hospital y sintomáticos que requieren apoyo mecánico ventilatorio. La tríada de síntomas son tos $(68 \%)$, fiebre $(89 \%)$ y disnea, esta última asociada a gravedad ( $92 \%)$; otros son fatiga $(38 \%)$, diarrea, cefalea, mialgias y anosmia o disgeusia $(30 \%)^{11}$. Los casos graves con SARS se definen como pacientes sintomáticos con dificultad respiratoria, frecuencia de 30 respiraciones por minuto, saturación de oxígeno < 93\% o índice de oxigenación presión arterial de oxígeno/ fracción inspirada de oxígeno $\left(\mathrm{PaO}_{2} / \mathrm{FiO}_{2}\right)$ menor de $300 \mathrm{mmHg}^{8}$, además alteraciones pulmonares serias $(41.8 \%)$ en la tomografía computarizada (TC). En una 
revisión de pacientes críticamente enfermos, las imágenes de TC revelaron opacidades en vidrio despulido $(87 \%)$, vidrio despulido más consolidación $(65 \%)$, agrandamiento vascular $(72 \%)$ y bronquiectasias $(53 \%)$, estas últimas tenían en el $87 \%$ de los casos distribución periférica, afectación pulmonar bilateral en el $82.2 \%$ y predominio pulmonar inferior o multifocal en el $54.5 \%$; comparativamente, los hallazgos de la radiografía de tórax mostraron opacidades reticular-nodulares bilaterales $(58 \%)$, opacidades en vidrio despulido $(48 \%)$, derrames pleurales $(33 \%)$, engrosamiento peribronquial $(25 \%)$ y consolidaciones focales $(20 \%)^{12}$. La fase inmunológica, donde la respuesta sistémica coloca al paciente en estado crítico, se presenta de 8 a 12 días de iniciada la enfermedad ${ }^{13}$; el compromiso multiorgánico se expresa por la alteración de las pruebas bioquími$\operatorname{cas}^{7}$ y se han descrito infartos de miocardio, miocarditis, trombosis pulmonar y choque séptico ${ }^{14}$.

\section{Efectos cardiovasculares de la infección por SARS-CoV-2}

La principal hipótesis sobre los efectos cardiovasculares es el endotelio; este órgano controla entre otros aspectos la inflamación, el estrés oxidativo, la permeabilidad vascular y el aporte energético a tejidos, y participa en la defensa inmunológica contra diferentes patógenos, generando inflamación significativa sistémica.

\section{Pericarditis/miocarditis}

El efecto proinflamatorio de las células endoteliales activadas por el patógeno expresan moléculas de adhesión leucocitaria (interleucina [IL] 1 alfa y beta, factor de necrosis tumoral alfa, moléculas de adhesión intercelular 1 y moléculas de adhesión vascular 1), producen reclutamiento leucocitario y tormenta de citocinas (IL-1 y ligando 10 de quimiocina con motivo C-X-C) a nivel miopericárdico ${ }^{15}$. El receptor por el que el virus accede a las células es el CD209, expresado en los macrófagos que promueven la invasión a tejidos vasculares y cardiacos ${ }^{16}$. La miocarditis aparece en casos severos de la enfermedad a los pocos días del inicio de la fiebre, lo que, aunado a la insuficiencia respiratoria, induce daño miocárdico con elevación de la enzima convertidora de angiotensina 2 en los vasos coronarios y en miocitos ${ }^{17}$. Esta entidad debe ser sospechada ante dolor precordial, cambios del segmento ST, arritmias, inestabilidad hemodinámica, niveles de troponina I y péptido natriurético tipo $B$ elevados y disfunción ventricular izquierda, dilatación e hipocinesia global. Comúnmente la enfermedad progresa a bloqueo en la conducción, taquiarritmias y disfunción ventricular progresiva, y se han reportado episodios de miopericarditis fulminantes ${ }^{18}$. El diagnóstico se confirma por resonancia magnética (RM) y biopsia endomiocárdica; histopatológicamente se han reportado necrosis de miocitos e infiltrados celulares mononucleares ${ }^{19}$. Una vez recuperados, estos pacientes requieren un periodo de reposo a fin de reducir el riesgo de insuficiencia cardiaca ${ }^{20}$, que varía entre tres y seis meses, basado en el análisis de la extensión de la inflamación y de función ventricular por RM con nivel de evidencia $2 A^{21}$.

\section{Vasculitis}

Los primeros casos de trombosis asociados a SARSCoV-2 fueron fundados ante la sospecha de vasculitis arterial neutrofílica. Los hallazgos histopatológicos muestran necrosis transmural arterial con infiltración masiva de neutrófilos en la media y adventicia, con raro involucro de la íntima ${ }^{22}$. La célula endotelial al ser activada por el patógeno libera endotelina 1 en respuesta a lipoproteínas de baja densidad oxidadas, trombina y angiotensina II, que genera la vasoconstricción, típica de la enfermedad vasculítica ${ }^{15}$; a esto sigue el depósito de complejos inmunitarios mediados (inmunoglobulina [lg] G, IgA e IgM) y fracciones de complemento, y suceden dos procesos principales en esta vasculitis, clasificada como hipersensibilidad tipo III: a) endotelitis aguda, donde las células endoteliales, habitadas por viriones, se infiltran de neutrófilos y elementos mononucleares, causando apoptosis, linfocitosis, eventos protrombóticos e inflamatorios, y b) peri/panarteritis, que es una infiltración de elementos inflamatorios, seguido de una cariólisis acelerada, acumulación de cuerpos apoptósicos y sustancias fibrinoides, indicando la presencia de una vasculitis leucocitoclástica. En otros casos la afectación es en grandes arterias (aortitis, aneurismas, hematomas intramurales o disecciones), y en niños se ha reportado enfermedad de Kawasaki. Estudios post mortem revelan daño alveolar difuso con congestión capilar pulmonar severa, y trombosis microvascular y macrovascular en corazón, intestino, riñones y pulmones ${ }^{23}$. Se ha descrito vasculitis urticarial, con exantema pruriginoso y rash petequial ${ }^{24}$.

\section{Síndrome coronario agudo}

Comúnmente el infarto agudo de miocardio suele ser el subtipo de afectación más común en el SARS y al 
síndrome respiratorio del Medio Oriente, y se ha hipotetizado la implicación de disfunción microvascular por infección de los pericitos como origen del infarto miocárdico sin enfermedad arterial obstructiva ${ }^{19}$. Ante un síndrome coronario agudo con elevación del ST se debe proceder a angioplastia primaria. Ante cambios atípicos del electrocardiograma (ECG), ya sea un caso confirmado o sospechoso de COVID-19, se recomienda el uso del ecocardiograma a fin de incluso descartar miocarditis ${ }^{25}$. Con concentraciones elevadas de troponina y cambios electrocardiográficos sugestivos de lesión incluso sin presentar elevación del segmento ST, pero en presencia de sintomatología sugestiva de infarto agudo de miocardio, los pacientes deben ser tratados bajo las recomendaciones de reperfusión, y todos los pacientes en esta condición deberán ser considerados y abordados como sospechosos de $\mathrm{CO}$ VID-1926. En todo caso, está indicado el envío a un programa de rehabilitación cardiovascular.

\section{Arritmias}

En Hubei (China) el $7.3 \%$ de los pacientes refirió palpitaciones como síntoma inicial ${ }^{27}$, en otro estudio el $16 \%$ de los casos presentaron arritmias, con mayor incidencia en pacientes en cuidados intensivos (44.4 vs. $6.9 \%)^{14}$. En 187 pacientes hospitalizados se reportó la incidencia de taquicardia y fibrilación ventriculares en el $5.9 \%^{28}$. También se ha informado presencia de bradicardia, bloqueos, taquicardia supraventricular y fibrilación auricular. La lesión del miocardio, miocarditis y procesos extracardiacos (sepsis, hipoxia, etc.) pueden exacerbar arritmias prexistentes por incremento en la demanda metabólica y la reducción de la reserva cardiaca $^{29,30}$, y otro factor ha sido el uso de medicamentos como hidroxicloroquina, lopinavir/ritonavir y azitromicina ${ }^{31}$.

\section{Eventos trombóticos}

Las anormalidades hemostásicas reportadas son trombocitopenia y elevación del dímero $\mathrm{D}$, las cuales se asocian a mayor necesidad de ventilación mecánica, manejo en terapia intensiva y mortalidad ${ }^{32}$, y hasta el $71 \%$ de los pacientes que mueren por COVID-19 cumplen con los criterios de la Sociedad de Trombosis y Hemostasia para coagulación intravascular disemina$\mathrm{da}^{33}$. La enfermedad crítica también se asocia a la prolongación del tiempo de protrombina y alto índice internacional normalizado; estas alteraciones pueden ser secundarias a la infección o como consecuencia de la tormenta de citocinas ${ }^{34}$. La anticoagulación, a pesar de evidencia científica no sólida, se basa en la opinión de expertos o en series muy pequeñas de pacientes ${ }^{35}$.

\section{Muerte súbita}

La muerte súbita (MS) no es una manifestación típica de la COVID-19 grave, sin embargo, se ha documentado MS en pacientes sin factores de riesgo y sin indicadores de mecanismos posibles de insuficiencia cardiaca ${ }^{36}$. Es posible que la propia infección, el estado inflamatorio, arritmias e hipoxia expliquen el cuadro; otras causas pueden ser fármacos que causan prolongación del QT y torsades de pointes $(\mathrm{TdP})$, o bien lo reportado en casos aislados con lopinavir/ritonavir, azitromicina y levofloxacina como causa de $\mathrm{TdP}^{37}$. Incluso la IL-6 parece causar prolongación del QT, bradicardia severa y bloqueos cardia$\cos ^{38,39}$. Un punto interesante es que la MS ocurre a pesar de la mejoría en las condiciones generales, de ahí la relevancia de evaluación de cada caso antes de iniciar ejercicio.

\section{Retorno al ejercicio población general}

\section{COVID-19 leve}

La mayoría de los casos de COVID-19 son de categoría leve y moderada, con duración de síntomas menor a seis semanas ${ }^{40}$, y se deberá reiniciar la actividad física una vez sea posible, para evitar efectos adversos del sedentarismo. Existe una amplia variación en la expresión del padecimiento y cada persona se recupera a un ritmo distinto ${ }^{41}$. Actualmente no existen algoritmos que guíen el regreso a ejercicio, pero en la pandemia por SARS-CoV-1 se reportó que la tolerancia al ejercicio y el estado de salud de los sobrevivientes al SARS fueron inferiores a los de la población general a seis meses ${ }^{42}$, además de que se documentaron alteraciones en la salud mental ${ }^{43}$. Lo anterior expresa situaciones donde el ejercicio es de gran beneficio, al cual se debe retornar de manera lenta, gradual y escalonada; no deben existir síntomas infecciosos y en caso de patología previa pulmonar o cardiovascular se deberá consultar al médico incluso si la enfermedad fue leve. De modo general, un paciente por lo demás sano con un curso autolimitado de COVID-19 que ha estado asintomático durante siete días puede comenzar a reanudar la actividad física al $50 \%$ del volumen previamente desarrollado, seguido de un 30,20 y $10 \%$ 
en las siguientes tres semanas si el paciente se siente cómodo y asintomático al final de cada semana; así conseguirá la readaptación, progresión y el reacondicionamiento de manera segura (Tabla 1).

\section{COVID-19 moderada}

El cuadro clínico moderado por COVID-19 puede durar hasta seis semanas, se caracteriza, además de los síntomas clásicos, por exacerbación del grado de disnea, fiebre persistente, tos y taquicardia ante leve actividad física, reportados entre los días 5 y 8 de iniciado el cuadro ${ }^{40}$; esto está causado por la mayor inflamación del parénquima pulmonar, pero sin llegar a presentar neumonía. El momento recomendado para el inicio del ejercicio es a siete días después de la resolución total de los síntomas ${ }^{44}$. Para iniciar un programa de ejercicio en este contexto, se recomienda hacerlo bajo la guía del médico que da seguimiento extrahospitalario al caso y sería idealmente en un programa de rehabilitación cardiopulmonar. Debido a la incertidumbre de la posible evolución y complicaciones después de la infección por SARS-CoV-2, se recomienda realizar una evaluación exhaustiva con historia clínica, amplia semiología de los síntomas actuales, exploración física y un ECG en reposo; idealmente, se debe contar con estudios de laboratorios, en especial los marcadores pronósticos de riesgo como troponina, una telerradiografía de tórax y una espirometría. Estudios más avanzados, como una prueba de esfuerzo, pruebas de función pulmonar y ecocardiograma transtorácico, serían ideales y ampliarían sin duda la información, pero no contraindican que se pueda iniciar el ejercicio. Las contraindicaciones para el ejercicio sería síntomas de novo, inestabilidad pulmonar o hemodinámica, o presencia de arritmias, lo cual implicaría suspender y reevaluar el caso de manera más profunda o referir a un centro de rehabilitación cardiopulmonar idealmente. En estos casos se recomienda seguimiento del experto.

\section{COVID-19 grave}

En la infección por SARS-CoV-2 existe un porcentaje alto de afección cardiaca además de la pulmonar ${ }^{10}$; por ende, el retorno al ejercicio idealmente debe estar indicado por un médico especialista en rehabilitación cardiopulmonar evaluando diferentes aspectos clínicos, de laboratorio y estudios de gabinete para documentar estabilidad médica justificado por el alto riesgo de eventos cardiovasculares mayores asociados a este virus tanto en fase aguda como en la convalecencia inclusive $^{45}$. El momento ideal es mínimo después de dos semanas de ausencia de síntomas, sin embargo, si se ha documentado daño miocárdico se deberá evitar ejercicio desde tres a seis meses, y con las condiciones siguientes.

\section{AsPectos ClíNICOS}

Es fundamental una historia clínica completa y semiología de los síntomas, el paciente deberá manifestarse idealmente asintomático por dos semanas; sin embargo, lo reportado después de COVID-19 más daño miocárdico es la presencia de astenia, adinamia, pérdida de peso y/o franca sarcopenia, deterioro de la capacidad funcional, disnea, debilidad generalizada, datos esperables después de un internamiento prolongado $^{46}$. Deberán estar ausentes cefalea, fiebre, dolor faríngeo, mialgias, artralgias, tos, dolor opresivo precordial, palpitaciones y moderada a importante intolerancia al esfuerzo. Naturalmente se deberá contar con estabilidad pulmonar (saturación de oxígeno > 90\%), presión arterial y frecuencia cardiaca en reposo dentro de los rangos normales.

\section{EstudIOS DE LABORATORIO}

Los biomarcadores deberán estar por debajo límite superior normal del percentil 99 que reporta cada laboratorio o bien tendiendo su curva hacia la normalización, especialmente la troponina, o en su defecto regresar a parámetros basales en caso de daño preexistente.

\section{Electrocardograma Y MONITOREO HOLTER}

Las manifestaciones eléctricas como arritmias y cambios dinámicos del segmento ST y onda $\mathrm{T}$, y cambios eléctricos de alto riesgo para choque cardiogénico y MS deberán estar controlados; los estudios deberán mostrar ausencia de estas anormalidades e idealmente documentar ritmo sinusal o mostrar cambios previos a la infección ya conocidos (ondas $\mathrm{Q}$, bloqueos de rama, etc.), o en su defecto con cambios producidos en el periodo agudo como necrosis miocárdica, pero tales hallazgos deben mantenerse estables en el seguimiento.

\section{ECOCARDIOGRAMA}

En el periodo agudo y grave se puede documentar disfunción global con alteraciones de la movilidad en 
Tabla 1. Ejercicio y deporte después de COVID-19

\begin{tabular}{|c|c|c|c|c|c|}
\hline & Cuadro COVID-19 & Días sin síntomas & Evaluación por experto & Estudios recomendados & Seguimiento \\
\hline \multirow{3}{*}{$\begin{array}{l}\text { Población } \\
\text { general }\end{array}$} & Asintomático y leve & $7-14$ & No & Ninguno & Recomendable \\
\hline & Moderado & $7-14$ & Sí & Rx Tx, ECG, EPM & Recomendable \\
\hline & Grave & $\begin{array}{l}>15 \\
90-180^{*}\end{array}$ & Sí & $\begin{array}{l}\text { MRs, EPM, ECG, H24, TCT, } \\
\text { ECO, PEAGE, RMNC* }\end{array}$ & Sí \\
\hline \multirow[t]{4}{*}{ Deportistas } & Asintomático & 14 & Sí & ECG & Sí \\
\hline & Leve & $14-28$ & Sí & MRs, ECG, ECO, PEAGE & Sí \\
\hline & Moderado & $14-28$ & Sí & $\begin{array}{l}\text { MRs, ECG, H24, ECO, TCT, } \\
\text { PEAGE }\end{array}$ & Sí \\
\hline & Grave & $\begin{array}{c}14-28 \\
90-180^{*}\end{array}$ & Sí & $\begin{array}{l}\text { MRs, ECG, H24, ECO, } \\
\text { PEAGE, TCT, RMNC** }\end{array}$ & Sí \\
\hline
\end{tabular}

*Ante afección miopericárdica.

Rx Tx: telerradiografía de tórax; ECG: electrocardiograma en reposo; EPM: espirometría; MRs: marcadores de riesgo en sangre; H24: Holter de 24 horas; TCT: tomografía computarizada de tórax; ECO: ecocardiograma transtorácico; PEAGE: prueba de esfuerzo con análisis de gases espirados; RMNC: resonancia magnética nuclear cardiaca.

todas las paredes o regionales, fracción de eyección del ventrículo izquierdo (FEVI) baja, derrame pericárdico e incluso disfunción ventricular derecha ${ }^{47}$, el ecocardiograma de control deberá mostrar mejoría en la función cardiaca global, ausencia o mínimo derrame pericárdico, y en general franca mejoría con respecto a los parámetros documentados durante la infección aguda en caso de contar con ellos, de lo contrario será de utilidad para valorar de dónde se parte y dar seguimiento a los hallazgos anormales ${ }^{48}$.

\section{Prueba de esfuerzo eléctrica/cardiopulmonar}

Son estudios fundamentales para una prescripción precisa del ejercicio. Ambos tipo de prueba darán información sobre la capacidad funcional medida por equivalentes metabólicos o consumo miocárdico de oxígeno calculado o directo, respectivamente, y la estabilidad hemodinámica ante el esfuerzo (respuesta presora, respuesta cronotrópica, arritmias, saturación de oxígeno en sangre). Solo mediante la prueba de esfuerzo con análisis de gases espirados podremos determinar el grado de afección pulmonar, cardiaca y muscular de cada paciente y dirigir de manera más específica el entrenamiento $^{49}$. Se deben tomar las medidas para evitar contagios durante este procedimiento ${ }^{50}$.

\section{RESONANCIA MAGNÉTICA NUCLEAR}

Es el estudio indicado ante COVID-19 complicada con miopericarditis. Ante este diagnóstico se aconseja un periodo de inactividad de tres a seis meses dependiendo de la evolución y los marcadores serológicos de riesgo. No obstante, debemos considerar un estudio donde se reportaron hallazgos diversos en pacientes no seleccionados recuperados de COVID-19, en el cual se encontró en el $78 \%$ algún grado de involucro cardiaco y en el $60 \%$ inflamación miocárdica activa, hallazgos independientes de las condiciones preexistentes, gravedad o curso de la infección aguda o el tiempo de diagnóstico ${ }^{51}$. Lo anterior deja abierta la necesidad de seguimiento a largo plazo para determinar las consecuencias clínicas de estos casos y refuerza la necesidad de hacer una evaluación completa antes de reiniciar ejercicio, con la amplia recomendación de que estos pacientes sean evaluados por médicos expertos en rehabilitación cardiopulmonar siguiendo los lineamientos de bioseguridad y clásicos de los programas.

\section{Deportistas}

Aunque los atletas no sean del grupo de riesgo para casos severos, la posibilidad de que se encuentre involucrado el corazón existe, incluso en asintomáticos, y es importante determinar si después de haber cursado con COVID-19 pueden ser elegibles para reanudar entrenamiento. Recordemos que el daño miocárdico, aunque en bajo porcentaje, complica la evolución ${ }^{52}$. La miocarditis es una causa de MS en menores de 35 años y los síntomas suelen ser inespecíficos (malestar general, mialgias, fatiga, bajo desempeño físico), por lo que en muchos casos no se sospecha, y el diagnóstico y la potencial miocarditis pueden ser pasados por alto. La evaluación ante sospecha de miocarditis puede 
ser confusa, por ejemplo, las alteraciones del ECG pueden atribuirse a adaptaciones por el entrenamiento y la elevación de troponina al ejercicio agudo; la poca especificidad de los síntomas, aunado a pruebas diagnósticas no claras, da por resultado una variedad de diagnósticos diferenciales. Con base en lo anterior, las recomendaciones de la Sociedad Europea de Cardiología, la Asociación Americana de Corazón y el Colegio Americano de Cardiología para el abordaje, detección y manejo de la miocarditis asociada a la COVID-19 es el siguiente ${ }^{53}$ :

- Atletas con COVID-19 asintomáticos: dado que en otros virus causantes de miocarditis la replicación viral se incrementa durante la actividad física vigorosa resultando en daño miocárdico adicional, se recomienda suspender toda actividad física intensa o competitiva por al menos dos semanas. En ausencia de síntomas y anormalidades en el ECG de reposo, al finalizar las dos semanas se podrá regresar al deporte sin restricción ${ }^{54}$.

- Atletas con COVID-19 sintomáticos sin evidencia de miocarditis: restricción de actividad física de dos a cuatro semanas. Se debe realizar una evaluación médica completa (historia clínica, examen físico, marcadores de riesgo, ECG, prueba de esfuerzo y ecocardiograma). El regreso al deporte se podrá realizar en ausencia de resultados anormales.

- Atletas con miocarditis: se prohibirá estrictamente toda actividad deportiva durante tres a seis meses, y el regreso al entrenamiento y competición se realizará una vez que se demuestre que la función ventricular izquierda y los diámetros cardiacos hayan regresado a la normalidad, que no existan arritmias relevantes y que los marcadores de inflamación e insuficiencia cardiaca se hayan normalizado ${ }^{55}$.

Se sabe que el pronóstico de los atletas que tuvieron miocarditis no complicada y se recuperaron completamente (FEVI normal y ausencia de reforzamiento tardío en RM) es altamente favorable. No obstante, se debe tener un seguimiento clínico para descartar complicaciones a largo plazo.

\section{Conclusiones}

La pandemia por COVID-19 ha generado hábitos higiénicos más conscientes y los cambios en el estilo de vida obligados; los cuadros clínicos variados y sus secuelas son retos para el retorno al ejercicio y deporte. La intervención multidisciplinaria en casos graves es regla, en especial la rehabilitación cardiopulmonar. Dada la evolución rápida de la información sobre la COVID-19, es posible que las orientaciones para el regreso seguro al ejercicio puedan presentar cambios, por lo que es necesario continuar atentos a la nueva información que sea publicada en el futuro.

\section{Agradecimientos}

Los autores desean expresar un profundo agradecimiento al Dr. Hermes Ilarraza Lomelí jefe del servicio de Rehabilitación Cardiaca del Instituto Nacional de Cardiología "Ignacio Chávez", maestro e impulsor de la investigación científica en el área de la cardiorehabilitación.

\section{Financiamiento}

\author{
Ninguno.
}

\section{Conflicto de intereses}

Los autores declaran no tener conflicto de intereses.

\section{Responsabilidades éticas}

Protección de personas y animales. Los autores declaran que para esta investigación no se han realizado experimentos en seres humanos ni en animales.

Confidencialidad de los datos. Los autores declaran que en este artículo no aparecen datos de pacientes.

Derecho a la privacidad y consentimiento informado. Los autores declaran que en este artículo no aparecen datos de pacientes.

\section{Bibliografía}

1. Leandro C, Ferreira E, Lima S. Covid-19 and exercise-induced immunomodulation. Neuroimmunomodulation. 2020;27:75-78.

2. Amatriain F, Gronwald T, Murillo R, Imperatori C, Solano A, Latini A, et. al. Physical exercise potentials against viral diseases like COVID-19 in the elderly. Front Med (Lausanne). 2020;7:379.

3. Nyenhuis S, Greiwe J, Zeiger J, Nannda A, Cooke A. Exercise and fitness in the age of social distancing during the COVID 19-pandemic. J Allergy Clin Immunol Pract. 2020:8:2152-5.

4. Blocken B, Malizia F, van Druenen T, Marchal T. Towards aerodynamically equivalent COVID $191.5 \mathrm{~m}$ social distancing for walking and running [Internet]. Euroga.org. Disponible en: https://www.euroga.org/system/1/ user_files/files/000/045/111/45111/150d3060c/original/Social_Distancing_v20_White_Paper.pdf

5. Jiménez $P$, Carbonell $B$, Lavie $C$. Physical exercise as therapy to fight against the mental and physical consequences of COVID-19 quarantine: Special focus in older people. Prog Cardiovasc Dis. 2020;63:386-8.

6. Baggish A, Levine B. Icarus and sports after COVID 19: Too close to the sun? Circulation. 2020;142:615-7.

7. Guan Wj, Ni Zy, Hu Y, Liang Wh, Ou Cq, He Jx, et al. Clinical characteristics of coronavirus disease 2019 in China. N Engl J Med. 2020;382:1708-20 
8. Wang T, Chau B, Lui M, Lam G, Lin N, Humber S. Physical medicine and rehabilitation and pulmonary rehabilitation for COVID 19. Am J Phys Med Rehabil. 2020;99:769-74.

9. Driggin E, Madhavan M, Bikdeli B, Chuich T, Laracy J, Biondi-Zoccai J, et al. Cardiovascular considerations for patients, health care workers, and health systems during the COVID-19 Pandemic. J Am Coll Cardiol. 2020;75:2353-71.

10. Zhou F, Yu T, Du R, Fan R, Liu Y, Liu Z, et al. Clinical course and risk factors for mortality of adults in patients with COVID-19 in Wuhan, China: a retrospective cohort study. Lancet 2020:395:1054-62

11. Brathia R, Marwaha S, Malhotra A, Iqbal Z, Hughes C, Börjesson M, et al. Exercise in the severe acute respiratory syndrome coronavirus-2 (SARS-CoV-2) era: A question and answer session with the experts endorsed by the section of Sports Cardiology \& Exercise of the European Association of Preventive Cardiology. Eur J Prev Cardiol. 2020;27:1242-51.

12. Arentz M, Yim E, Klaff L, Riedo F, Chong M, Lee M, et al. Characteristics and outcomes of 21 critically ill patients with COVID-19 in Washington State. JAMA. 2020;28:1612-14.

13. Huang $C$, Wang $Y$, Li X, Ren L, Zhao J, Hu Y. Clinical features of patients infected with 2019 novel coronavirus in Wuhan, China. Lancet. 2020; 395:497-506

14. Wang D, Hu B, Hu C, Zhu F, Liu X, Zhang J. Clinical characteristics of 138 hospitalized patients with 2019 novel coronavirus-infected pneumonia in Wuhan, China. JAMA. 2020;323:1061-9.

15. Libby P, Lüscher T. COVID-19 is, in the end, a endothelial disease. Eur Heart J. 2020:41:3038-44.

16. ESC Guidance for the Diagnosis and Management of CV Disease during the COVID-19 Pandemic [Internet]. European Society of Cardiology [Fecha última actualización: 10 de junio de 2020]. Disponible en: https:// www.escardio.org/Education/COVID-19-and-Cardiology/ESC-COVID-19-Guidance

17. Chen C, Zhou Y, Wang DW. SARS-Cov-2: a potential novel etiology of fulminant miocarditis. Herz. 2020:45:230-2.

18. Liu Y, Yang Y, Zhang C, Huang F, Wang F, Yuan J, et al. Clinical and biochemical indexes from 2019-Cov infected patients linked to viral loads and lung injury. Sci China Life Sci. 2020;63:364-74.

19. Guzik T, Mohiddin S, Dimarco A, Patel V, Savvatis K, Marelli-Berg F, et. al. COVID-19 and the cardiovascular system: implications for risk assessment, diagnosis, and treatment options. Cardiovasc Res. 2020;116:1666-87.

20. Barker D, O'Sullivan O, Senaratne K, Baker P, Cranley M, Dharm D, et.al. The Stanford Hall consensus statement for post-COVID-19 rehabilitation. Br J Sports Med. 2020:54:949-59.

21. Schellhorn $\mathrm{P}$, Klingel K, Burgstahler K. Return to sports after COVID 19-infection. Eur Heart J. 2020;41(46):4382-4.

22. Klok FA, Kruip MJHA, van der Meer NJM, Arbous MS, Gommers DAMPJ, Kant KM, et al. Incidence of thrombotic complications in critically ill ICU patients with COVID-19. Tromb Res. 2020;191:145-7.

23. Menter T, Haslbauer J, Nienhold R, Savic S, Hopfer H, Deigendesch N, et al. Postmortem examination of COVID-19 patients reveals alveolar damage with severe capillary congestion and variegated findings in lungs and other organs suggesting vascular dysfunction. Histopathology. 2020;77:198-209

24. Becker R. COVID-19 associated vasculitis and vasculopathy. J. Thromb Thrombolysis. 2020;50:499-511.

25. Mahmud E, Dauerman H, Welt F, Messenger J, Rao S, Grines S, et. al. Management of acute myocardial infarction during the COVID-19 pandemic. A Consensus Statement from the Society for Cardiovascular Angiography and Interventions, the American College of Cardiology and the American College of Emergency Physicians. Catheterization and Cardiovascular Interventions. Catheter Cardiovasc Interv. 2020; 96:336-45.

26. Ibanez B, James S, Agewall S, Antunes M, Bucciarelli D, Bueno H, et al. 2017 ESC Guidelines for the management of acute myocardial infarction in patients presenting with ST-segment elevation: The Task Force for the management of myocardial infarction in patients presenting with ST-segment elevation of the European Society of Cardiology. Eur Heart J 2018;39:119-77.

27. Liu K, Fang Y, Deng Y, Deng Y, Li W, Wang M, et. al. Clinical characteristics of novel coronavirus cases in tertiary hospitals in Hubei province. Chin Med J. 2020:133:1025-31.

28. Guo T, Fan Y, Chen M, Wu X, Zhang L, He T, et al. Cardiovascular implications of fatal outcomes of patients with coronavirus disease 2019 (COVID-19). JAMA Cardiol. 2020;5:1-8.

29. Xiong T, Redwood S, Prendergast B, Chen M. Coronaviruses and the cardiovascular system: acute and long-term implications. Eur Heart $\mathrm{J}$. 2020;41:1798-800.

30. Bhatla A, Mayer M, Adusumalli S, Hyman M, Oh E, Tierney A, et al. COVID-19 and cardiac arrhythmias. Heart Rhythm. 2020;17;1439-44.
31. Saenz L, Miranda A, Speranza R, Alkmim R, Enriquez A, Figuereido M, et al. Recommendations for the organization of electrophysiology and cardiac pacing services during the COVID-19 pandemic: Latin American Heart Rhythm Society (LAHRS) in collaboration with: Colombian College Of Electrophysiology, Argentinian Society of Cardiac Electrophysiology (SADEC), Brazilian Society Of Cardiac Arrhythmias (SOBRAC), Mexican Society Of Cardiac Electrophysiology (SOMEEC). J Interv Card Electrophysiol. 2020;29:1-7.

32. Bikdeli B, Madhavan M, Jimenez D, Chuich T, Dreyfus I, Driggin E, et al. COVID-19 and thrombotic or thromboembolic disease: Implications for prevention, antithrombotic therapy, and follow-up. J Am Coll Cardiol. 2020;75:2950-73.

33. Levi M, Toh C, Thachil J, Watson H. Guidelines for the diagnosis and management of disseminated intravascular coagulation. British Committee for Standards in Haematology. Br J Haematol. 2009;145:24-33.

34. Lippi G, Plebani M, Henry B. Thrombocytopenia is associated with severe coronavirus disease 2019 (COVID-19) infections: a meta-analysis. Clin Chim Acta. 2020;506:145-8.

35. Vivas D, Roldán V, Esteve P, Roldan I, Tello M, Ruíz N, et al. Recommendations on antithrombotic treatment during the COVID-19 pandemic. Position statement of the Working Group on Cardiovascular Thrombosis of the Spanish Society of Cardiology. Rev Esp Cardiol. 2020;73:749-57.

36. Inciardi R, Lupi L, Zaccone G, Italia L, Raffo M, Tomasoni D, et al. Cardiac involvement in patients with cornavirus disease 2019 (COVID 19). JAMA Cardiol. 2020;5:1-6.

37. Shirazi S, Mami S, Mohtadi N, Ghaysouri A, Tavan H, Nazari A, et al. Sudden cardiac death in COVID-19 patients, a report of three cases. Future Cardiol. 2021;17(1):113-8.

38. Lazzerini P, Boutjdir M, Capecchi L. COVID-19, arrhythmic risk and inflammation: mind the gap! Circulation. 2020;142:7-9.

39. Azarkish M, Laleh F, Eslami M, Mollazadeh R. Transient complete heart block in a patient with critical COVID-19. Eur Heart J. 2020:41:2131

40. Metzl J, McElheny K, Robinson J, Scott D, Sutton K, Toresdahl B. Considerations for return to exercise following mild-to-moderate COVID-19 in the recreational athlete. HSS J. 2020;16(Suppl 1):1-6.

41. Verwoert G, de Vries S, Bijsterveld N, Willems A, Borgh R, Jongman J, et al. Return to sports after COVID-19: apposition paper from the Dutch Sports Cardiology Section of the Netherlands Society of Cardiology. Neth Heart J. 2020;28:391-5.

42. Hui D, Joynt D, Wong K, Gomersall D, Li T, Antonio G, et al. Impact of severe acute respiratory syndrome (SARS) on pulmonary function, functional capacity and quality of life in a cohort of survivors. Thorax. 2005;60:401-9.

43. Tansey M, Louie M, Loeb M, Gold W, Muller M, De Jager J, et al. Oneyear outcomes and health care utilization in survivors of severe acute respiratory syndrome. Arch Inter Med. 2007;167:1312-20.

44. Caterisano A, Decker D, Snyder B, Feigenbaum M, Glass R, House P, et al. CSCCa and NSCA joint consensus guidelines for transition periods: safe return to training following inactivity. Strength Cond J. 2019;41:1-23.

45. Long B, Brady W, Koyfman A, Gottlieb M. Cardiovascular complications in COVID-19. Am J Emerg Med. 2020;38:1504-07.

46. Morley J, Kalantar Z, Anker S. COVID-19: a major cause of cachexia and sarcopenia? J Cachexia Sarcopenia Muscle. 2020;11:863-5

47. Rodriguez S, Minguito C, Echarte M, Del Castillo G, Valdivia R, Fernández V. Echocardiographic findings in critical patients with COVID-19. Rev Esp Cardiol. 2020;73:849-64.

48. Shi S, Qin M, Shen B, Cai Y, Liu T, Yang F. Association of cardiac injury with mortality in hospitalized patients with COVID-19 in Wuhan, China. JAMA Cardiol. 2020;5:802-10.

49. Herdy A, Ritt L, Stein R, Araújo C, Milani M, Meneghelo R, et.al. Cardiopulmonary exercise test: Background, applicability and interpretation. Arq Bras Cardiol. 2016:107:467-81.

50. Venturelli M, Cè E, Paneronis M, Guazzi M, Lippi G, Paoli C, et al. Safety procedures for exercise testing in the scenario of COVID-19: a position statement of the Società Italiana Scienze Motorie e Sportive. Sport Sci Health. 2020;16:601-7.

51. Puntmann V, Carerj M, Wieters I, Fahim M, Arendt C, Hoffmann J, et al. Outcomes of cardiovascular magnetic resonance imaging in patients recently recovered from coronavirus disease 2019 (COVID-19). JAMA Cardiol. 2020;5(11):1265-73

52. Yeo TJ. Sport and exercise during and beyond the COVID-19 pandemic. Eur J Prev Cardiol. 2020;27:1239-41.

53. Pelliccia A, Solbert E, Papadakis M, Adami P, Biffi A, Caselli S, et al. Recommendations for participation in competitive and leisure time sport in athletes with cardiomyopathies, myocarditis and pericarditis: position statement of the Sport Cardiology Section of the European Association of Cardiology Section of the European Association of Preventive Cardiology. Eur Heart J. 2019;40:19-33.

54. Löllgen H, Bachi N, Papadopoulou T. Recommendations for return to sport during the SARS-CoV-2 pandemic. BMJ Open Sp Ex Med. 2020;6:e000858.

55. Phelan D, Kim J, Chung E. A game plan for the resumption of sport and exercise after coronavirus disease 2019 (COVID-19) infection. JAMA Cardiol. 2020:5:1085-6. 